\title{
Multiscale structure in sedimentary basins
}

\author{
S. A. Stewart,* G. J. Hay, † P. L. Rosin $\ddagger$ and T. J. Wynn§ \\ *BPAzerbaijan, Sunbury on Thames, Middlesex, UK \\ †Université de Montréal, Montréal, Québec, Canada \\ $\ddagger$ Cardiff University, Cardiff, UK \\ §TRACS International, Union Grove, Aberdeen, UK
}

\begin{abstract}
Hierarchies of superimposed structures are found in maps of geological horizons in sedimentary basins. Mapping based on three-dimensional (3D) seismic data includes structures that range in scale from tens of metres to hundreds of kilometres. Extraction of structures from these maps without $a$ priori knowledge of scale and shape is analogous to pattern recognition problems that have been widely researched in disciplines outside of Geoscience. A number of these lessons are integrated and applied within a geological context here. We describe a method for generating multiscale representations from two-dimensional sections and 3D surfaces, and illustrate how superimposed geological structures can be topologically analysed. Multiscale analysis is done in two stages generation of scale-space as a geometrical attribute, followed by identification of significant scalespace objects. Results indicate that Gaussian filtering is a more robust method than conventional moving average filtering for deriving multiscale geological structure. We introduce the concept of natural scales for identifying the most significant scales in a geological cross section. In three dimensions, scale-dependent structures are identified via an analogous process as discrete topological entities within a four-dimensional scale-space cube. Motivation for this work is to take advantage of the completeness of seismic data coverage to see 'beyond the outcrop' and yield multiscale geological structure. Applications include identifying artefacts, scale-specific features and large-scale structural domains, facilitating multiscale structural attribute mapping for reservoir characterisation, and a novel approach to fold structure classification.
\end{abstract}

\section{INTRODUCTION}

The term 'scale' refers to the spatial dimensions at which entities, patterns and processes can be observed and measured. From an absolute perspective, scale corresponds to a standard system, such as cartographic scales and census units, used to partition geographical space into operational spatial units. In a relative framework, scale is a variable intrinsically linked to the entities under observation, and corresponds to ones' window of perception. Thus every scale reveals information specific to its level of observation (Marceau, 1999). Scale is composed of two fundamental parts: resolution and extent. Resolution refers to the smallest intervals in an observation set (i.e. the sample interval or grid spacing), while extent refers to the range over which observations at a particular resolution are made (i.e. the area of interest) (Hay et al., 2001). In this study, small scale refers to a small area, and large or coarse scale represents a large area.

Subsurface mapping has traditionally been based on projection of surface geology, constrained by sparse sub-

Correspondence: S. A. Stewart, BP Azerbaijan, c/o Chertsey Road, Sunbury on Thames, Middlesex TW16 7LN, UK. E-mail: stewarsal@bp.com surface data points from drilled wells and mines. But the amount of detail observed at exposures is typically much greater than that conveyed by stratum contours, which tend to connect subsurface control points by smooth curves until they abut at faults (Tearpock \& Bischke, 2002). Mappers tend to intuitively accept this variation in structural architecture at different scales of observation. For example, use of fractals to characterise fault populations and topographic surfaces is well documented (Cowie et al., 1995; Bonnet et al., 2001), but spatial variations of fractal characteristics are not widely studied (e.g. Xu et al., 1993; Belfield, 1998; Veneziano \& Iacobellis, 1999). Various schemes that assign 'order' to structures at different scale have been devised in field studies (e.g. Fleuty, 1964; Ramsay, 1967), but are restricted to manual interpretation of cross sections and not widely employed. This paper pursues the idea of structural order by demonstrating automated methods of identifying scale-dependent structure in two and three dimensions within large data sets of digital geological mapping.

Seismic reflection data, three-dimensional (3D) seismic data in particular, introduces an additional and fundamentally different control on subsurface mapping. The main difference in relation to sparse data types is provision of a continuous set of control points on a relatively closely 


\section{S. A. Stewart et al.}

spaced 3D grid (typically around $10 \mathrm{~m}$ ). Geological surfaces mapped using these data capture structure at a wide range of scales. At small scales, structures are truncated by the sampling resolution of the grid (tens of metres), and at large scales by the size (i.e. area of interest) of the survey $(10-100 \mathrm{~km})$. The data density and coverage of seismic data fills the 'gap' between outcrop and regional scale structure. This is particularly evident in sedimentary basins, where individual surfaces - bedding planes or their lateral correlatives - can be mapped with confidence for tens of thousands of square kilometres, capturing far more structural information than yielded by interpolation of drilled well control points or projection of sporadic surface outcrop. In these settings, a range of different scales of observation can reveal a hierarchy of scale-dependent structural forms. Investigation of data at multiple scales is known as multiscale or multiresolution analysis (Mokhtarian \& Mackworth, 1992; Finkelstein \& Salesin, 1994). Consideration of multiscale structure raises both general and specific questions related to structural geology. For example:

- How can superimposed geological structures of different scales be mapped and measured?

- To what extent are outcrop-scale rock properties like fracture permeability determined by larger-scale structures that may be unseen at outcrop or in the wellbore?

- Should fold-classification schemes be qualified with scale-dependent terms?

- Are interference and parasitic fold patterns (often considered to be genetically unrelated and related fold systems respectively) end-members of a geometric continuum?

Although relatively little has been published on scale-dependent structure in a geological context, the concept of scale has been investigated and employed in other disciplines including financial time series analysis (Zhang et al., 2001), signal processing (Gammaitoni et al., 1998), computer vision (Lindeberg, 1999), video compression (Mokhtarian et al., 2002), landscape ecology (Hay et al., 2001) remote sensing (Marceau \& Hay, 1999), biomedical engineering (Sajda et al., 2002) and the social and natural sciences (Marceau, 1999). The objective of this paper is to show how some of the techniques developed in these other disciplines can be brought to bear on geological structure, and begin to address questions such as those previously defined.

This paper begins with examples of seismic-based mapping in sedimentary basins to demonstrate variation in geological structure through scale. This is followed by a review of methods for two-dimensinal (2D) analysis of scale-dependent structure, followed by description of a method for automatically identifying 'natural scales' in geological cross sections. The method is then generalised to three dimensions - an example of scale-space analysis of a geological surface mapped on 3D seismic data illustrates a discussion of the topology of scale-space in sedimentary basins. We conclude with description of potential applications and note that the tools discussed here may facilitate exploration of a relatively novel subdiscipline, multiscale structural geology.

\section{TYPICAL SEISMIC IMAGING OF A SEDIMENTARY BASIN}

Figure 1 illustrates reflection seismic profiles from the North Sea basin (Fig. la, b) and the south Caspian basin (Fig. 1c-e). In each case, small- and large-scale structure is illustrated by comparison of the zoom-ins with the large-scale (regional) sections. In both basins, different structural features, picked out by curvature of the seismic reflectors, are apparent at different scales. We focus on folds and minor faults in this study because faults with large throw are treated as steep zones in a gridded surface whereas in reality, they are breaks in surface continuity and should ideally be excluded from the analysis by structural restoration. Figure 1 illustrates a number of challenges facing any technique devised to extract scale-dependent geological structure from reflection seismic data:

- To operate on the basis of no a priori knowledge of the scale and form of the geological structure.

- Fold structures at a given scale might be individual and localised, rather than members of continuous fold sets.

- At small scales there will be a noise threshold associated with data acquisition.

- The technique should be applicable in 2D (i.e. sections) and 3D (surfaces).

\section{RESAMPLING, DECOMPOSITION AND SMOOTHING}

When working with high-resolution data sets such as detailed digital elevation models or a subsurface horizon mapped on 3D seismic data, several numerical techniques are available to tackle the challenge of finding large-scale structure, though the techniques are not necessarily widely used for this purpose. In the following section, we discuss the strengths and weaknesses of the principal techniques, in order to provide a context for the choice of algorithm used in this study.

\section{Resampling}

2D profiles and 3D surfaces are routinely resampled from an original grid spacing of 12.5 or $25 \mathrm{~m}$ to 50 or $100 \mathrm{~m}$, by a process loosely termed decimation. Decimation is a form of generalisation that is generally applied to reduce the quantity of data for the sake of decreasing software runtime and computer disk usage. The procedure usually involves selecting every second, fourth or eighth data point (corresponding to 25,6 or $1.6 \%$ of a gridded surface). The data between the resampled points becomes redundant unless an operation such as smoothing has been applied prior to resampling. Decimation also takes advantage of 


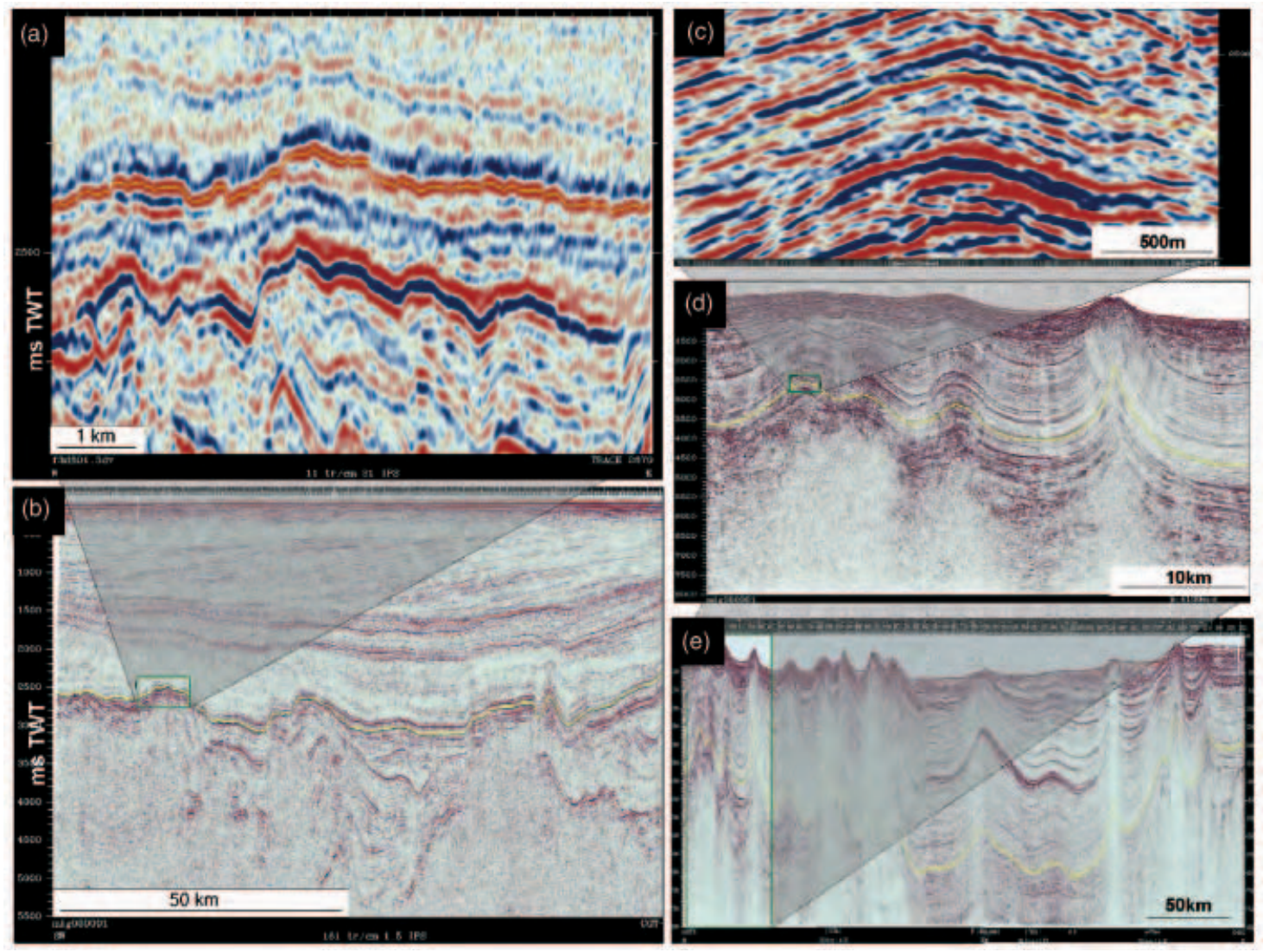

Fig. 1. Reflection seismic examples (2D profiles) of scale-dependent structure from two sedimentary basins. (a, b) from the central North Sea show a mixture of geological structure and seismic noise at sub-kilometre scale (a), and structures up to basin curvature at a scale of hundreds of kilometres (b). (c-e) show the relatively simple, kilometre-scale fold train of the south Caspian Sea. Additional structures are seen within the folds (c) and at basin scale (e).

the constraint that a regular sampling interval can record wavelengths no shorter than twice the sample spacing (e.g. Telford et al., 1976, pp. 376-378) - so the more decimated the data set, the smoother the stratum contours. However, decimation also gives rise to aliasing, which is the creation of nonreal structure as a by-product of being unable to capture real features that have wavelengths shorter than double the sampling interval. An example of aliasing in a subsurface map is illustrated in Fig. 8 of Stewart \& Podolski (1998).

Bergbauer \& Pollard (2003) reject decimation as a mechanism for removing high frequencies, on the basis of aliasing. An experiment published by Bergbauer \& Pollard (2003, their Fig. 11) is reproduced here (Fig. 2a, b), demonstrating how progressive undersampling of an arbitrary analytical function produces distortion (i.e. aliasing) of the original form. This distortion is initially manifest as changes in the position and shape of fold hinges, until finally the original form becomes unrecognisable (Fig. 2a). The onset of aliasing depicted in Fig. 2a is reflected in attributes such as curvature (Fig. 2b).

An alternative approach to decimation involves a 'moving window' where the data points are sampled for attribute calculation at some desired sampling interval. Unlike decimation as described above, this process is repeated at each data point within the initial high-resolution data set, so the derived data has the same number of data points as the original data set (minus some data points around the margins of the surface where the sampling window half-width exceeds the distance to the data set edge). The moving-window approach is less prone to aliasing associated with the sampling frequency because the sampling is repeated at every grid node in the initial data set (Stewart \& Podolski, 1998; Belfield, 2000; Stewart \& Wynn, 2000). Figure 2c illustrates the results of curvature measurements at the same resampling intervals used in Fig. $2 \mathrm{~b}$, and demonstrates that the aliasing problems such as fold-hinge misposition are much reduced.

\section{Decomposition}

Fourier transforms are widely used for decomposing continuous signals into their component frequencies, and have been applied to geological fold shape (Stabler, 1968; Ramsay \& Huber, 1987; Stowe, 1988), geomorphological surfaces (Pike \& Rozema, 1975) and bedding surfaces mapped on 3D seismic in sedimentary basins (Bergbauer et al., 2003). Gallant \& Hutchinson (1997) critically discussed applying Fourier spectral analysis to regional geological surfaces. They pointed out that Fourier harmonics are continuous within an area of interest; consequently, their properties are independent of location, in contrast 


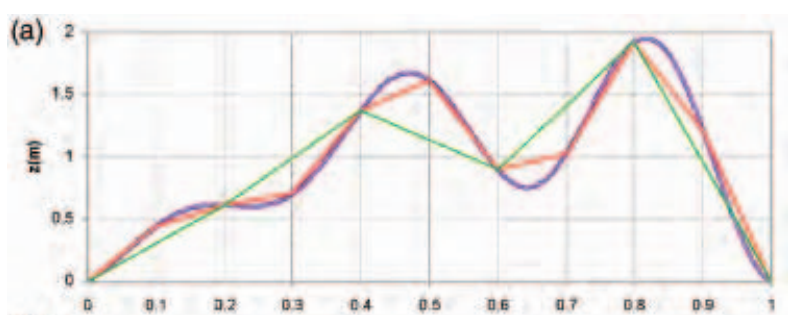

(b)

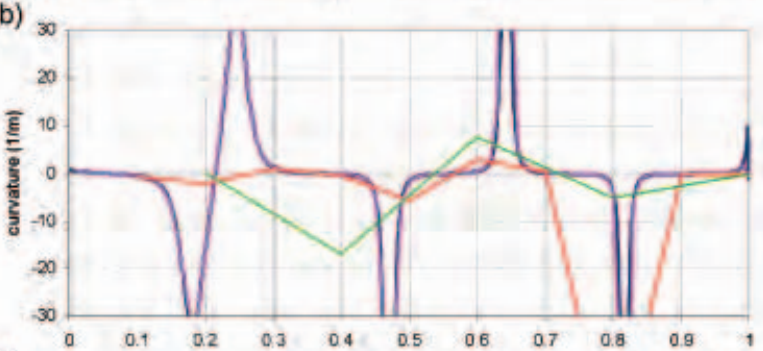

(c)

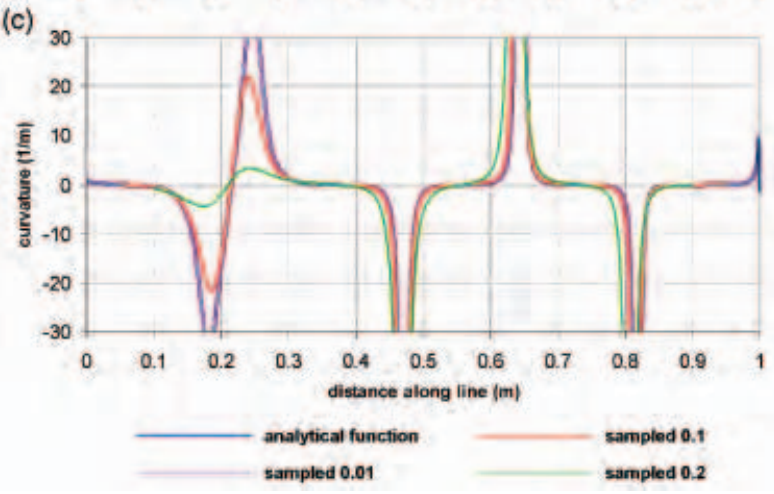

Fig. 2. Numerical experiment showing the effects of sampling and aliasing, partly after Bergbauer \& Pollard (2003). (a) An arbitrary curved line (dark blue) is progressively sampled by fewer and fewer points. Below a sampling interval that is significant with respect to the main structures (Nyquist frequency), the sampled data quickly loses the original signal. (b) As the sampling interval becomes wider, structural attributes such as curvature bear less relationship to attributes calculated on the original, analytical data. (c) Curvature calculations of part (b) repeated using a moving window approach. Although there are inaccuracies, aliasing is much reduced.

to geological structures that are often localised, isolated features. Secondly, harmonics are sine functions that may not adequately represent the complex geological forms in the source data. Finally, nonsinusoidal geological shapes produce short-wavelength harmonics that overwhelm the contribution of smaller geological features at these wavelengths. Conversely, Bergbauer et al. (2003) obtained promising results by removing frequency bands in the Fourier domain to produce low-cut or high-cut filtering, but it was not clear how this method could be automated to isolate the most significant harmonics. Bergbauer et al. (2003) also tested the performance of singular-value decomposition and factorial kriging; both yielded poorer results than Fourier filtering.

Wavelet transforms represent a family of techniques for multiscale signal decomposition that have theoretical advantages over Fourier transforms for geological mapping. In particular, the basis function is discrete rather than continuous. However, defining the form of the function remains a challenge. Positive wavelets have been used to decompose topographic surfaces into a number of 'features' at different scales (Gallant \& Hutchinson, 1997). As with Fourier transforms, however, these features are fixed in shape and the validity of structural attributes derived from them, or a partially decomposed surface, is open to question. Anisotropic and 'complex' wavelets with variable directional properties exist (Darrozes et al., 1997; Kingsbury, 1999), and may overcome this problem, but have yet to be applied to geological mapping.

\section{Smoothing}

Smoothing, or fairing, is perhaps the most obvious approach to removing high-frequency components, and is a routine step in producing maps from reflection seismic data (e.g. Roberts, 2001). Several published studies of scale-dependent geological structure have been based on smoothing (e.g. Ericsson et al., 1998; Bergbauer \& Pollard, 2003). For most seismic interpreters, however, degree of smoothing tends to be driven by map aesthetics rather than explicit consideration of frequency content, and there is limited choice of smoothing filters (or adequate reason for selecting one technique over another) in most commercial seismic interpretation software. Review of image-processing literature reveals that a wide range of smoothing algorithms exist, ranging from a simple moving average filter, through various weighted averages, to more sophisticated edge-preserving algorithms and feature detectors (e.g. Gonzalez \& Woods, 2002). In the following sections, we compare the equally weighted moving average filter and Gaussian filtering within the context of generating multiscale geological structure.

\section{The moving average filter}

A moving average filter, where all data points are equally weighted within a user-specified window (box-car filter) is probably the most commonly used smoothing device in

Fig. 3. Results of smoothing of the Caspian regional marker shown in Fig. le. (a) Original profile (thick red line) overlain by 250 versions that are smoothed by moving average within progressively wider windows, from 1 to $125 \mathrm{~km}$ window width (data point spacing $250 \mathrm{~m}$ ). Arrows highlight examples of lateral migration of fold-hinge locations as a function of smoothing. Circles show anomalous short wavelength features (Slutzky Effects) in smoothest profile. (b) Map view of surface derived from profiles in (a). $x$-axis is the cross-section length $(300 \mathrm{~km}) y$ axis is width of smoothing window $(0-125 \mathrm{~km})$ and can be thought of as the smoothing, or scale, 'dimension'. The unsmoothed profile (lowermost (red) in part (a)) is placed along the $x$-axis, the smoothest (topmost (yellow) in part (a)) is at the top of the map. (c) Perspective view of surface shown in (b). (d-f) Repeat of the first three displays, this time with Gaussian smoothing. The $y$-axis in the map and perspective view corresponds to Gaussian filter $\sigma$. 
Multiscale geological structure

(a)

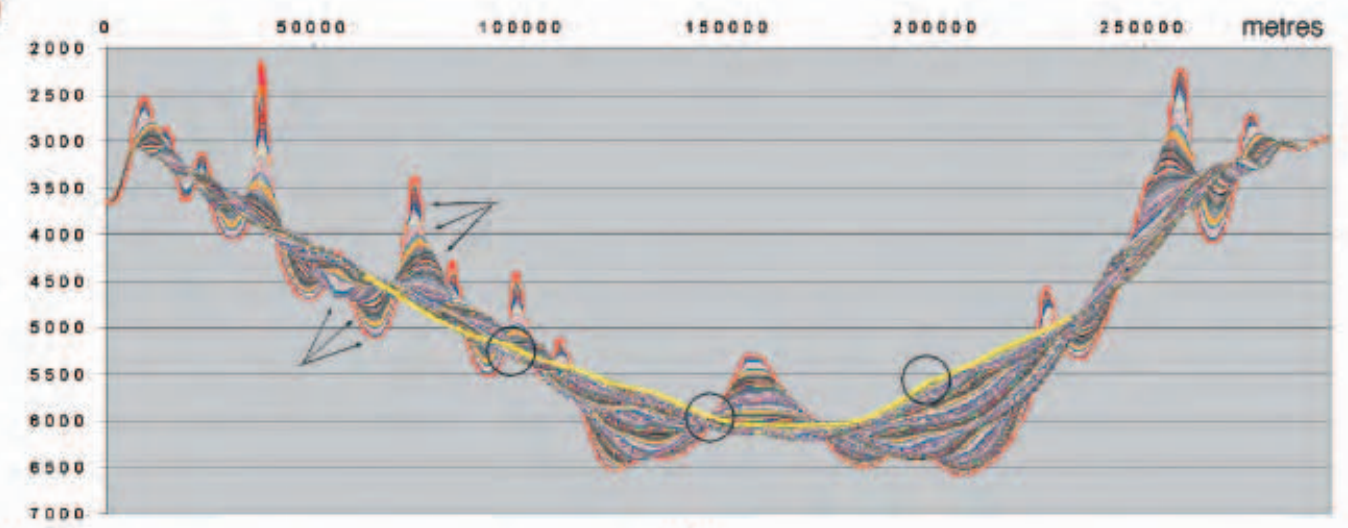

(b)

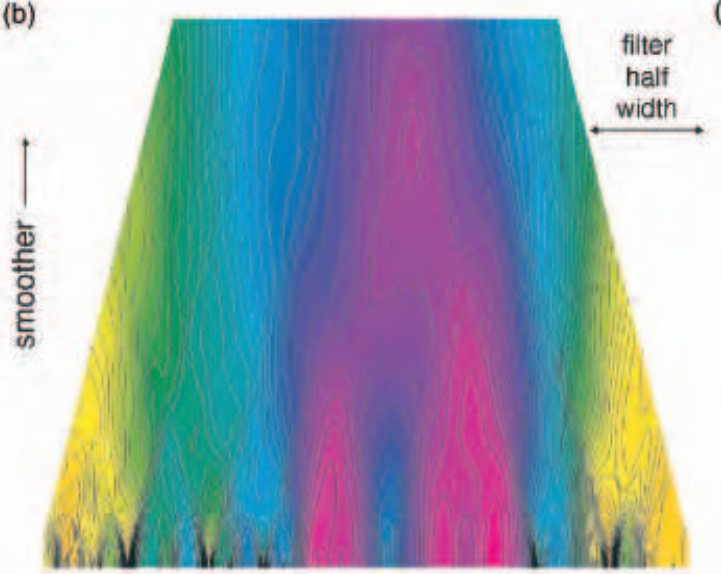

(c)

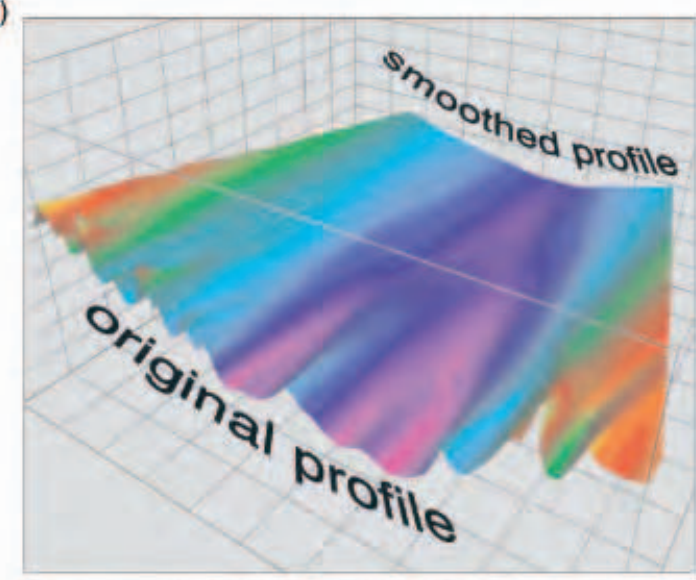

(d)

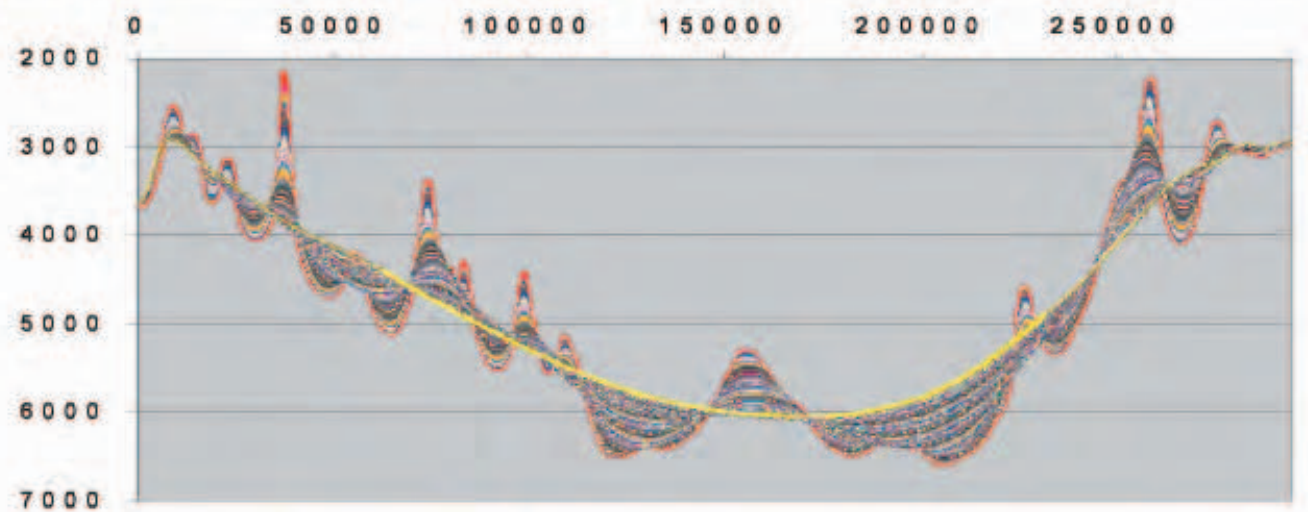

(e)

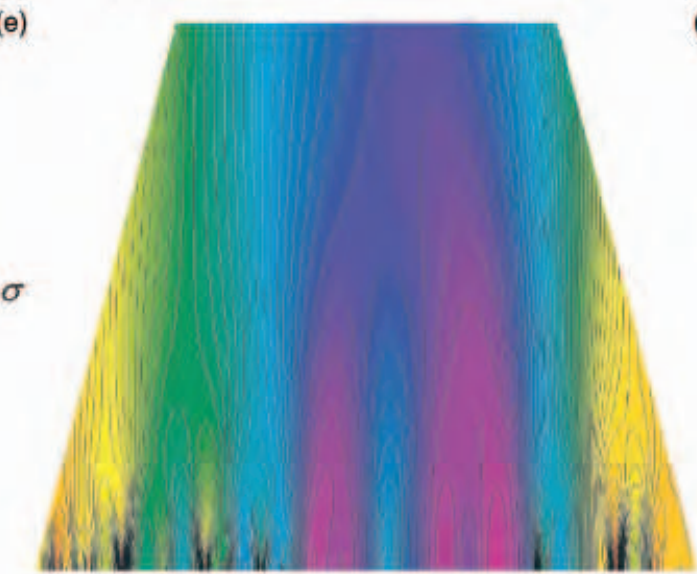

(f)

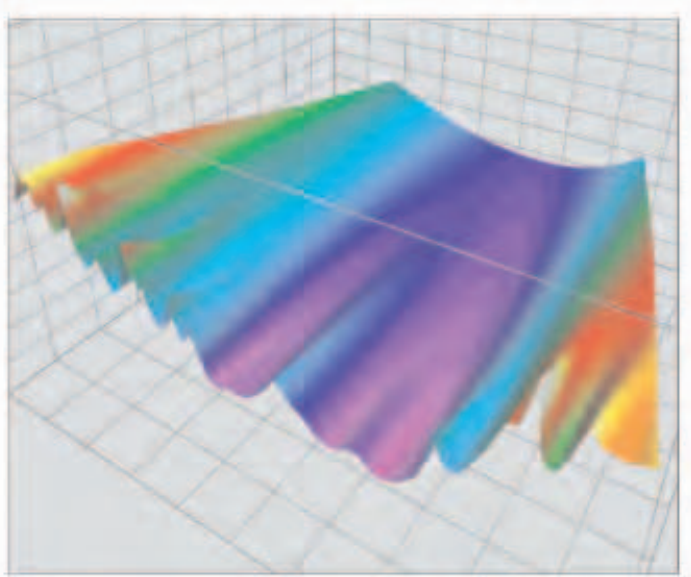


geological mapping (e.g. Bergbauer \& Pollard, 2003). It can be shown, however, that equal weighting is less effective than a weighted mean for the purposes of eliciting scaledependent structure. Figure 3 shows the effect of applying moving average smoothing to the folded regional marker shown in Fig. le. In this experiment, the original 2D profile was smoothed by a moving average filter with 250 different kernel sizes (kernel is an arrangement of numbers that constitute a filter). The narrowest filter was three data points wide (a $1 \times 3$ kernel resampling a $500-\mathrm{m}$ window) and the resulting curve is plotted immediately on top of the original profile in Fig. 3a. This result is then superimposed by the results from the other 249 kernel sizes, culminating in the widest $(1 \times 501$ kernel resampling a $125-\mathrm{km}$ window) and represents the topmost profile plotted (in bold yellow) on Fig. 3a. The consecutively smoother versions of the original surface can also be used to construct a 3D surface to help visualise the scale dimension, or scalespace (Fig. 3b, c). The lateral migration (mispositioning) of fold hinges noted in the resampling example (Fig. 2a) is seen once again with progressive moving average smoothing (Fig. 3a-c). The most heavily smoothed profile (yellow in Fig. 3a) shows that relatively short wavelength structure has persisted, or been created, in spite of the aggressive level of smoothing. These phenomena are known as Slutzky effects after an economist who showed that a moving average might generate an irregular oscillation even if none exists in the original data (Slutzky, 1937).

\section{Gaussian filtering}

An alternative to the arithmetic mean is some form of weighted average, either linear or more complex. In a discussion of scale dependence in topography, Wood (1996) suggested that distance-dependent weighting should be user-defined (but criteria were not specified). An approach commonly employed in image and signal processing is weighting according to a Gaussian function (Eqn. (1) defines a one-dimensional Gaussian filter).

$$
G(x)=\frac{1}{\sigma \sqrt{2 \pi}} e^{-x^{2} / 2 \sigma^{2}}
$$

where $x$ is distance from the median point of the filter and $\sigma$ is the standard deviation that defines the shape (width) of the weighting profile. Widespread usage of Gaussian smoothing reflects a number of advantageous properties, including, spatial shift invariance (i.e. no preferred location of filter), thus all locations are measured in the same fashion. Isotropy (i.e. no preferred orientation of filter), and no new structures are created in the transition from fine to coarse scale. Babaud et al. (1986), Weickert (1997), Lindeberg (1999) and Hay et al. (2002) discuss Gaussian filtering in more detail and conclude that this family of smoothing operators is a good choice for multiscale analysis - especially without a priori information. Figure $3 \mathrm{~d}-\mathrm{f}$ repeat the smoothing exercise of Fig. $3 \mathrm{a}-\mathrm{c}$, this time convolving the data representing the regional marker from
Fig. le with a Gaussian filter through a range (1-250) of standard deviations $(\sigma)$. Figure 3d compared with Fig. 3a shows that the most smoothed (uppermost) profile is free from the high-frequency artefacts produced by moving average filtering. The relative stability of Gaussian smoothing is particularly clear in the comparison of map views of the surfaces that represent the progressively smoothed profile (Fig. 3b, e, c, f). Lateral migration of fold hinges is less pronounced. Given the widespread use of Gaussian smoothing in other disciplines and the relatively good performance of this approach to smoothing demonstrated here, Gaussian smoothing is adopted here as the core process for investigating scale-dependent geological structure. Figure 3e can be viewed as a scale-space representation of the original 2D geological profile (Witkin, 1984), and is the basis of the natural scales analysis in the next section.

\section{D MULTISCALE GEOLOGICAL STRUCTURE - NATURAL SCALES}

The 3D surfaces generated by applying a Gaussian filter to smooth a single 2D profile in Fig. 3 show that structure appears to evolve progressively with smoothing, rather than in a series of abrupt steps, raising the question: with no a priori knowledge of the scale of the geological structural elements, how can we identify specific geological frequencies in the initial data, given the continuous nature of scale-space? To address this question, we introduce the concept of natural scales, which seeks to identify real structures within a scale-space curve or profile, according to some significance criterion, while excluding the redundant information (Bengtsson \& Eklundh, 1991; Rosin, 1992, 1998).

\section{A technique for determining natural scales}

Multiscale analysis requires two main components: the generation of a multiscale representation, and a feature detector. Given a scale-space representation of a profile (Fig. 3e), the problem of detecting natural scales becomes the task of subdividing scale-space according to structural significance. The technique employed here was described in a review of methods for calculating natural scales by Ro$\sin (1992)$, which is built on the notion that structures can be identified by changes in the sign of curvature. This is conceptually the same condition used by Ramsay (1967, pp. 351-355) to identify hierarchies of median surfaces in fold trains (Fig. 4a). The primary weakness of the median surface method was that a given order of median surface could contain structure at a wide variety of scales (Fig. 4b, c), therefore could not isolate scale-specific structure.

Curvature is independent of the overall reference frame of the profile, so delimiting structures by inflections, or 'zero-crossings' of curvature, rather than changes from concave up to concave down, has the advantage of rotation invariance. In practice, the second derivative gives similar, 


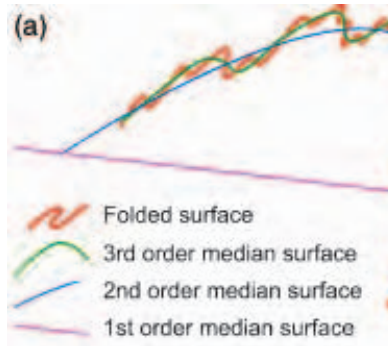

(a)

(b)

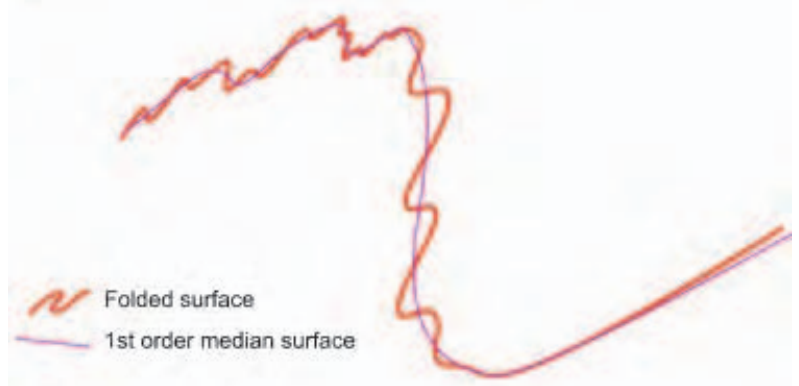

(c)

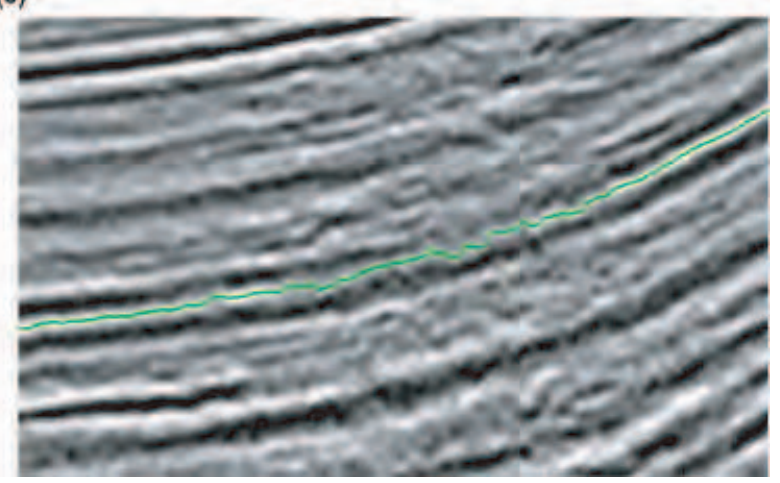

Fig. 4. Defining structural 'order' in a folded surface using median surfaces (after Figure 7-11 of Ramsay, 1967). (a) Each median surface is a minimum curvature spline connecting the curvature 'zero-crossings' in the lower-order surface. (b) Showing how spatial variation in structural scale in the initial data is reflected in the median surface, raising the question, how can median surfaces in themselves isolate scale-dependent structure? (c) Typical piece of seismic data from a sedimentary basin showing variations in roughness of autopicked surface due to variations in data quality - these would translate into spatial variation in structure of median surfaces.

if less robust, results in profiles from sedimentary basins (Roberts, 2001). Rather than working with the scale-space data set itself (e.g., Fig. 3e), we use the scale-space plot of the zero-crossings of curvature (Mokhtarian \& Mackworth, 1992). Also known as a 'curvature scale-space image', this plot is constructed by recording the location of curvature zero-crossings with increasing degree of Gaussian smoothing. The curvature scale-space image generated from the profile in Fig. le is shown in Fig. 5a. The arch-shaped areas enclosed by curvature zero-crossings in scale-space can be thought of as scale domains that define scale-dependent geometry of the individual struc- (a)

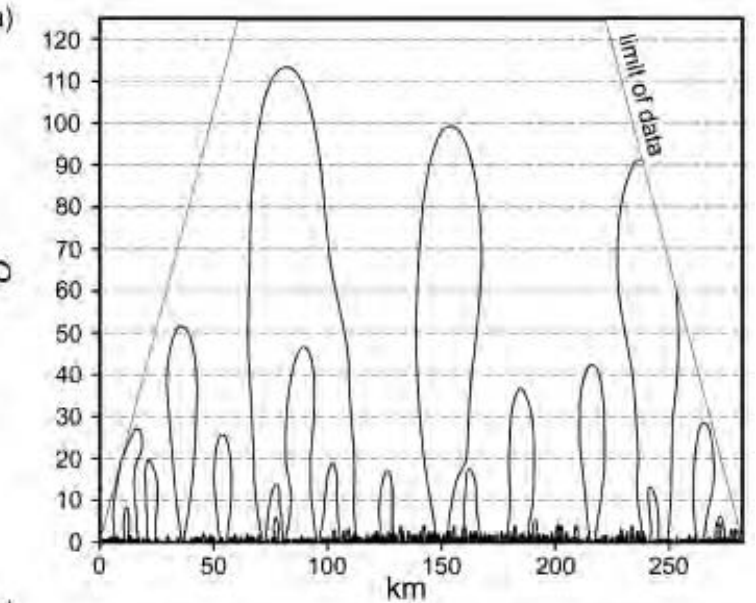

(b)
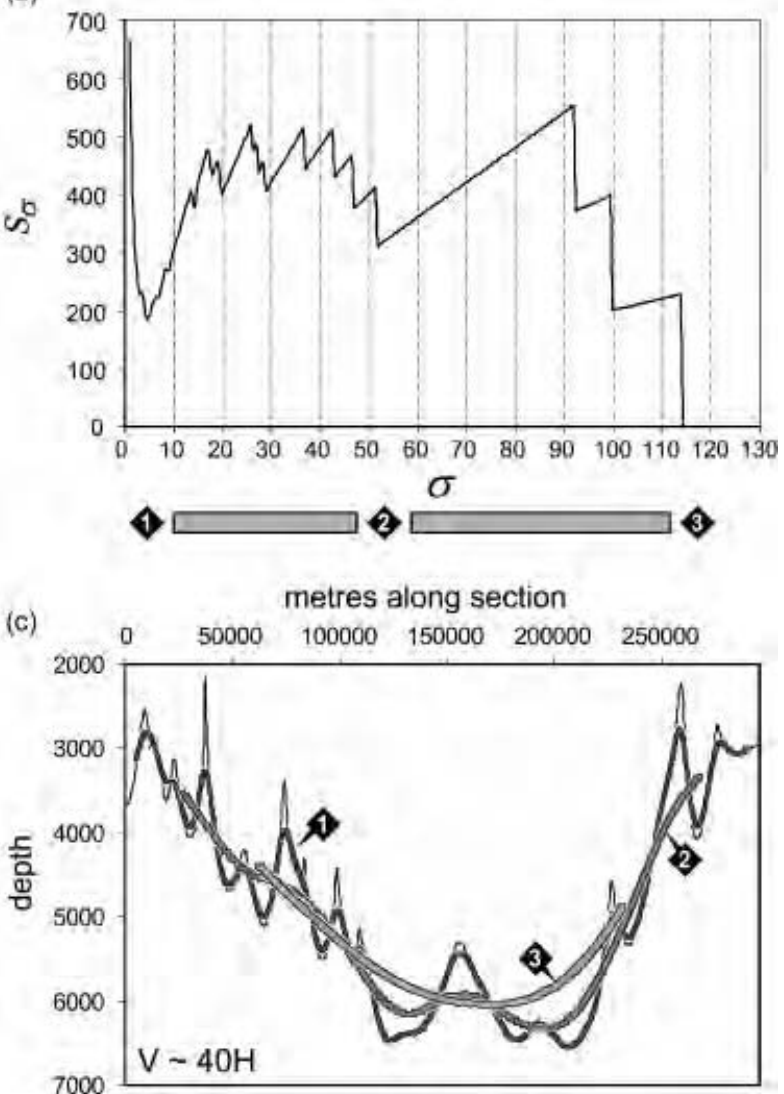

Fig. 5. Natural-scale analysis of Fig. 1e. (a) Curvature scale-space image of the profile highlighted in Fig. 1e. Axes are same as in Fig. $3 \mathrm{e}$. Arches represent position of curvature zero-crossings in $\sigma-$ profile length space. The standard deviation units are the same as the sampling interval of the geological profile, in this case $250 \mathrm{~m}$, so at $\sigma=40,1$ standard deviation is $10 \mathrm{~km}$ from the median point. (b) Significance measure $\left(S_{\sigma}\right)$ against $\sigma$. Local minima represent natural scales, lumped here into three groups. The minima could equally have been split to represent individual structures.

Diamond shapes identify scales representing structural order. (c) The structural orders identified in (b) displayed as the corresponding smoothed versions of the original profile. The original profile is displayed as the thin, lowermost line. This is a subset of the profiles in Fig. 3d. 


\section{S. A. Stewart et al.}

tures they enclose. Arch width is an indication of structural wavelength (distance along the original profile with constant curvature sign after a given amount of smoothing), and height is a measure of the scale. Structures do not persist (in scale) beyond the crest of their enclosing arches.

The density of zero-crossings as a function of scale is then used to identify 'natural scales'. In our definition, natural scales are defined by step changes in the density of zero-crossings. Visual inspection of Fig. 5a suggests the presence of several natural scales within the original profile. The population of the shortest wavelength structures exists at $\sigma<5$, a loose group of intermediate scale structures are removed when $15<\sigma<50$, and three larger structures dominate the interval $90<\sigma<110$. Beyond $\sigma=110$ only the basin scale structure remains, though it does not appear on Fig. 5a as there are no associated inflections in the curve representing the basin (Fig. 3d). We test here a numerical method of identifying these scale-specific groups, using a significance measure $\left(S_{\sigma}\right)$. At each scale, $S_{\sigma}$ is defined as the sum of the number of zero-crossings of curvature at all points on the curve normalised by the Gaussian smoothing scale $\sigma$ (Fig. 5b). Natural scales are defined to be at scales producing local minima of $S_{\sigma}$, where individual structures or groups of structures disappear (Bengtsson \& Eklundh, 1991; Rosin, 1992). According to this criterion, Fig. 5b shows three defined orders of structure: the lowest (1) corresponds to the individual folds seen in Fig. 1e, the highest (3) is the basin scale curvature seen in Fig. 3e. In Fig. 5c we illustrate the results of applying Gaussian filtering to the original profile at these natural scales, to reveal optimised orders of structure.

The natural scales shown in Fig. 5 are global in the sense that a smoothing operator of a given scale is convolved with all of the original data but the result will only yield structure where it exists in the original data, based on specific threshold criteria. Nonetheless, there are several techniques available for calculating natural scales locally rather than globally (review by Rosin, 1998). However, calculation of local natural scales tends to be less stable than the global method, and is not further pursued in this study.

\section{Discussion of natural scales}

The results in Fig. 5 show that natural-scale analysis of a fold belt with a regional data set can isolate smooth forms of individual fold structures, and at the largest scale, detect basinal curvature that is difficult to measure in the initial data. The first natural scale effectively removes the highest frequency noise in the data/interpretation, but preserves the wavelength and amplitude characteristics of the major fold structures. This low-order natural scale could be used in curvature analysis to determine strain distribution and related reservoir parameters (e.g. Ericsson et al., 1998; Roberts, 2001; Hart et al., 2002). The higher-order, largerscale structures are not obvious within spatially restricted subsets of data, but could contribute to the finite strain within the deformed layers and could also be the subject of curvature analysis to factorise scale-specific structure from the total strain at outcrop or well scale (Stewart \& Wynn, 2000). An alternative approach would be to use a high-order natural scale as a structural restoration template to remove basin curvature (for example) before strain analysis. The difference between the first natural scale and
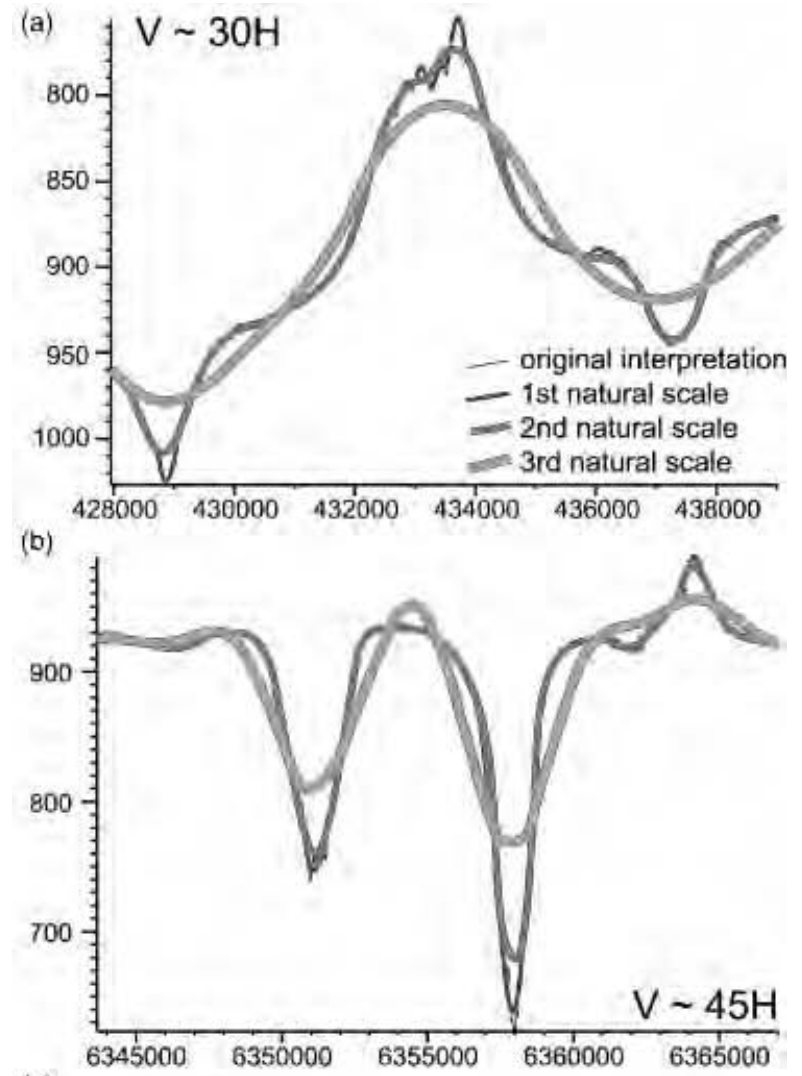

(c)
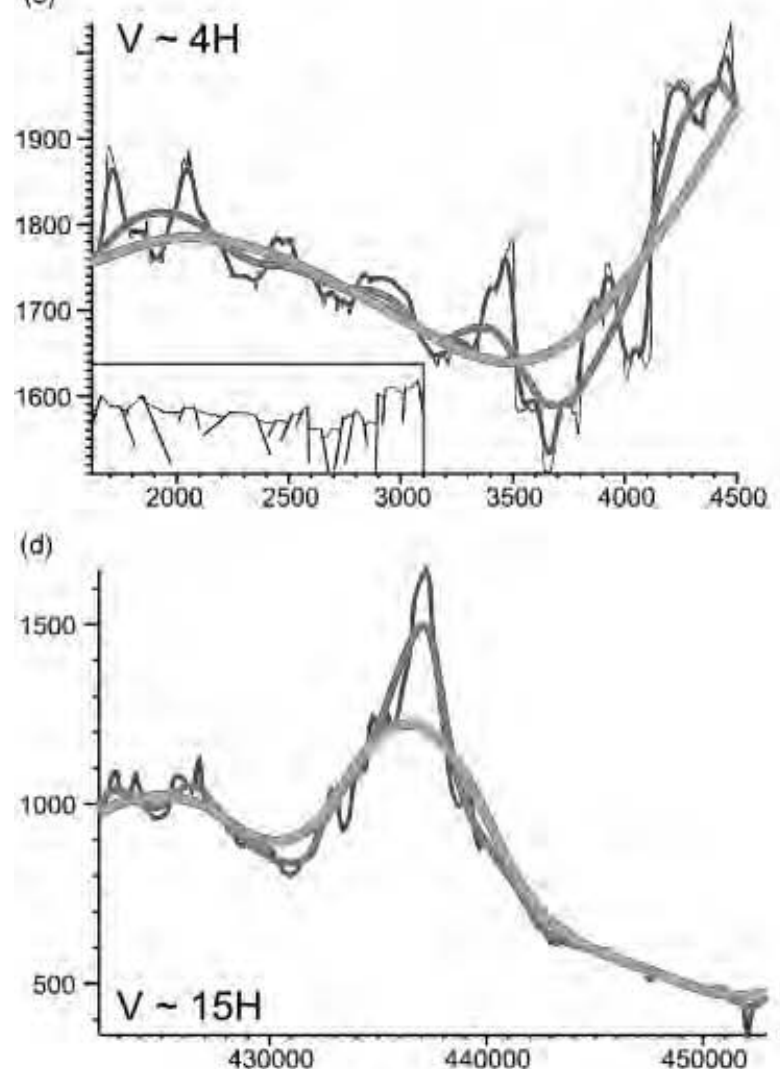

C 2004 Blackwell Publishing Ltd, Basin Research, 16, 183-197 
the original data, or between low-order natural scales is the equivalent of applying a low-cut filter and can reveal, for example, sedimentary features by removing local fold structures (e.g. Fig. 8 of Carter, 2003).

Gaussian filtering reduces the area enclosed by the curve or surface (e.g. Lowe, 1989). This 'shrinkage' affects closed curves (e.g. diapirs) more severely than open ones (e.g. regional bedding planes). It can be measured and corrected by various methods, such as normalising the integrals of the original and smoothed curves (Desbrun et al., 1999). The Fig. 3d examples show a maximum shrinkage of $3.5 \%$ (at $\sigma=125$ ). The examples in Fig. 6 include a shrinkage correction method described by Lowe (1989).

Several different geological data sets were used to test the method further. The results are illustrated in Fig. 6. Figure 6a represents a stratigraphic marker overlying a salt diapir in the central North Sea. The first natural scale is difficult to distinguish from the initial data and retains rugosity due to local degradation in seismic data quality. The second natural scale removes this rugosity but preserves the overall form of the drape fold, and some adjacent deepwater channels. The third scale emphasises the wavelength of the large features in the surface but does not represent the shape of these structures accurately. Figure $6 \mathrm{~b}$ is a section through two deepwater channel cuts. The natural scales yield the same information as discussed for Fig. 6a, but it is now clearer that the third natural scale is a poor representation of the structural form even though the wavelength is accurately measured. It is also evident that the structural amplitude is suppressed in spite of having been corrected for shrinkage. The examples so far have been free from abrupt changes in dip.

Faulted surfaces, on the other hand, challenge all methods of analysing scale-dependent structure. The main problems are the sharp edges at fault cutoffs and the spatial gaps in structure between the fault cutoffs. Figure $6 \mathrm{c}$ shows a faulted surface in which interpretation of the regional marker is connected by interpretation along the fault planes, giving a combination of sharp edges, fault planes and unfaulted strata within the fault blocks. The natural-scale surfaces treat the whole data set as a continuous curve and do not distinguish between the fault planes and unfaulted strata. Solving this issue is beyond the scope of this paper, though there are some obvious geometrical possibilities such as restricting natural-scale analysis to strata within major fault blocks, or doing natural-scale analysis on a surface with the major faults restored. Figure $6 \mathrm{~d}$ shows natural-scale analysis of a topographic profile

Fig. 6. Natural scales in various cross-sections. Each example shows the initial profile as a thin black line overlain by the first three natural scales. Axis labels in metres, all have significant vertical exaggeration (labelled). (a) Drape fold overlying North Sea diapir. (b) Deep water channel cuts in North Sea Miocene surface. (c) Faulted intra-Permian surface, southern North Sea. Inset shows original interpretation at $\mathrm{V}=\mathrm{H}$, with faults marked. (d) Present day topographic profile across the sub-Andean foothills, Bolivia. from a present day mountain range. In this case, natural scales isolate the position and areal extent of the principal topographic features.

In each of the cases shown in Fig. 6, as in Fig. 5, manual input was required at the stage of deciding whether to 'lump or split' the natural scales detected by $S_{\sigma}$ minima. So the technique involves an element of interpretation and is best described as 'semi-automatic'. Nonetheless, it represents a step forward in the structural analysis of seismic interpretation by objectively identifying scales of interest that can go forward into further attribute analysis. Although the analysis has been on 2D sections rather than 3D surfaces so far, $2 \mathrm{D}$ analysis can represent a rapid, automated guide to determine the degree of smoothing to be applied to large surface data sets prior to further analysis (e.g. curvature measurement). The next section of this paper describes application of a similar technique to 3D surfaces.

\section{D MULTISCALE GEOLOGICAL STRUCTURE - SCALE-SPACE TOPOLOGY}

Multiscale analysis of a 3D surface is done in two stages similar to the approach to analysing $2 \mathrm{D}$ profiles - generation of scale-space, followed by feature detection. Several methods are available along these lines (Hay et al., 2003). We generate 'linear scale-space' (Lindeberg, 1994; Hay et al., 2002) to provide a multiscale representation of a geological surface mapped on 3D reflection seismic data (i.e. a gridded surface, or digital elevation model), and employ 'blob-feature detection' (Hay et al., 2002) for automatically defining dominant multiscale components from this representation.

\section{Linear scale space}

Scale-space is an uncommitted framework for early visual operations that was developed by the computer vision community to automatically analyse real-world structures at multiple scales - specifically, when there is no a priori information about these structures, or the appropriate scale(s) for their analysis (Lindeberg, 1994). 'Uncommitted framework' refers to observations made by a front-end vision system (i.e. an initial-stage measuring device) such as the retina, a camera or a reflection seismic survey that involves no knowledge, and no preference for anything. When no scale information is known about a map or visual scene, the only reasonable approach for an uncommitted vision system is to represent the input data at (all) multiple scales. The example chosen here to test this method in a geological context is a patch of $3 \mathrm{D}$ seismic interpretation from a surface overlying a salt diapir in the Central North Sea, that is known to contain a variety of types of geological and artefact structure at various scales (Fig. 7a, b).

In practice, surface elevation is treated as a height attribute, enabling the surface to be converted to a greyscale image, or map. Gaussian filters are applied to this initial 


\section{S. A. Stewart et al.}

greyscale image at a range of kernel sizes resulting in a scale-space cube or stack of progressively smoothed image layers, where each new image layer represents convolution at an increased scale (Fig. 7c). This is a process similar to the smoothing of $2 \mathrm{D}$ profiles discussed earlier in this pa- per (Fig. 3c-e), though here, we are using the zeroth-order derivative of a '2D' Gaussian function (Eqn. (2)).

$G(x, y)=\frac{1}{2 \pi \sigma^{2}} e^{-\left(\left(x^{2}+y^{2}\right) / 2 \sigma^{2}\right)}$ (a)

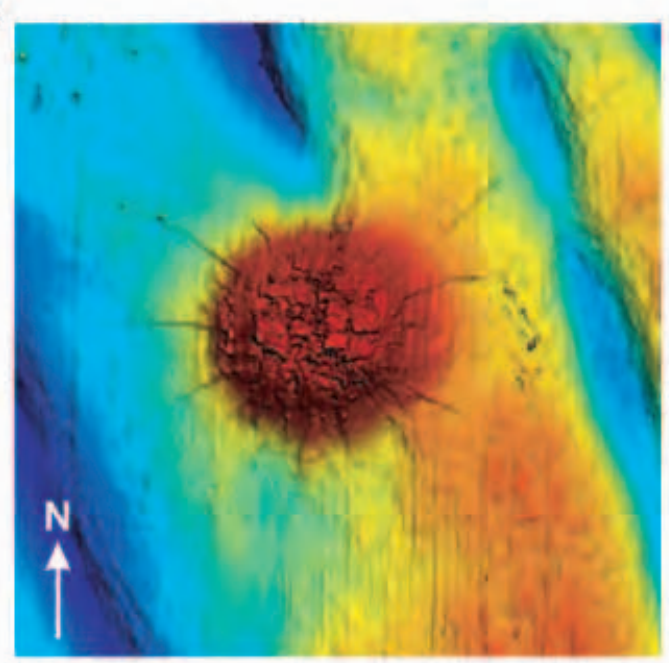

(c)

(d)

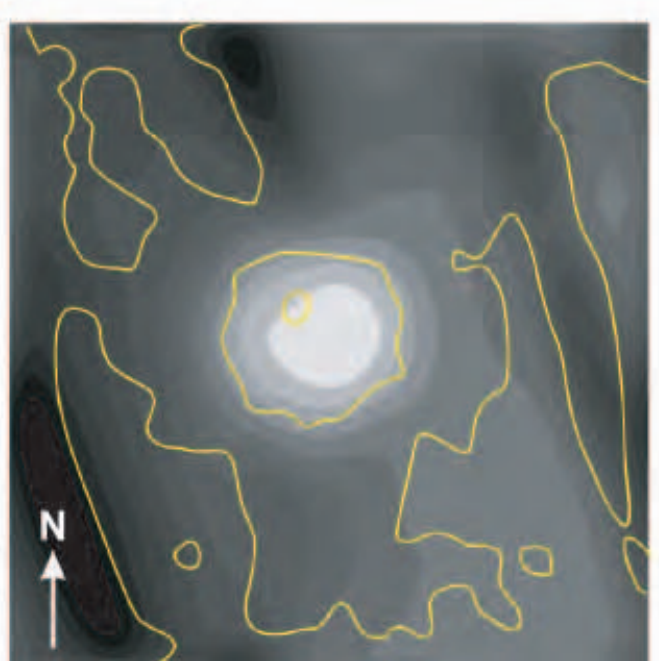

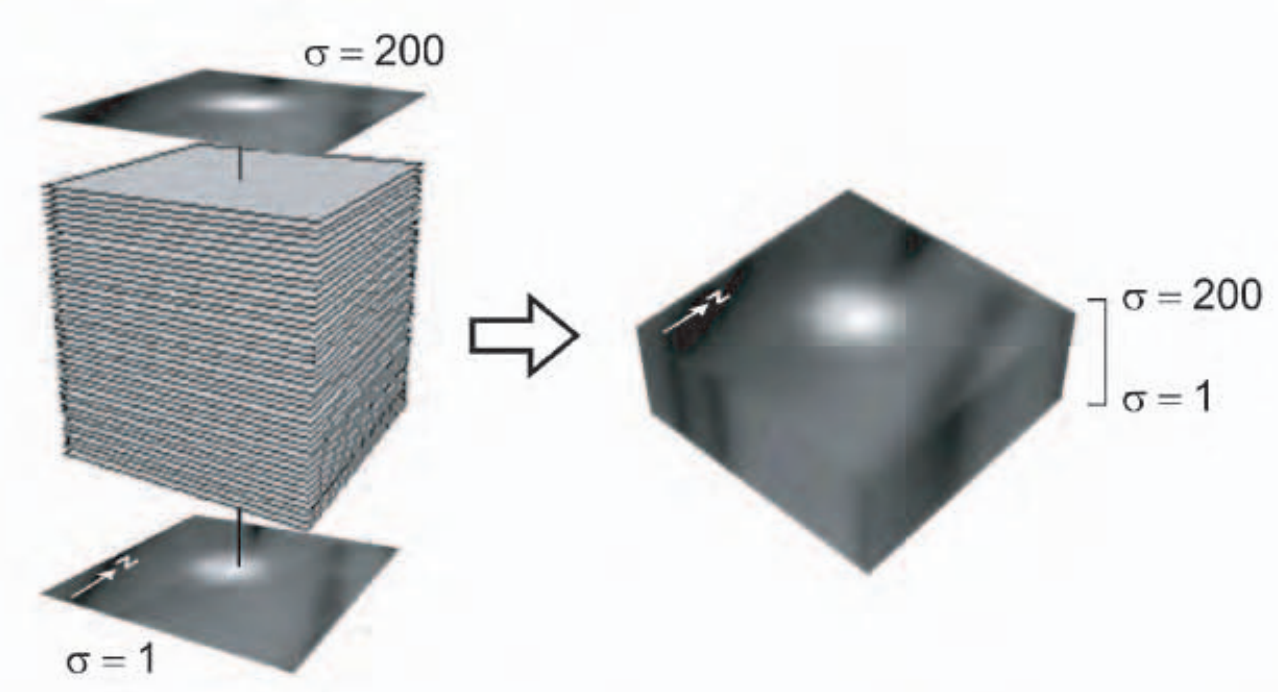

(b)

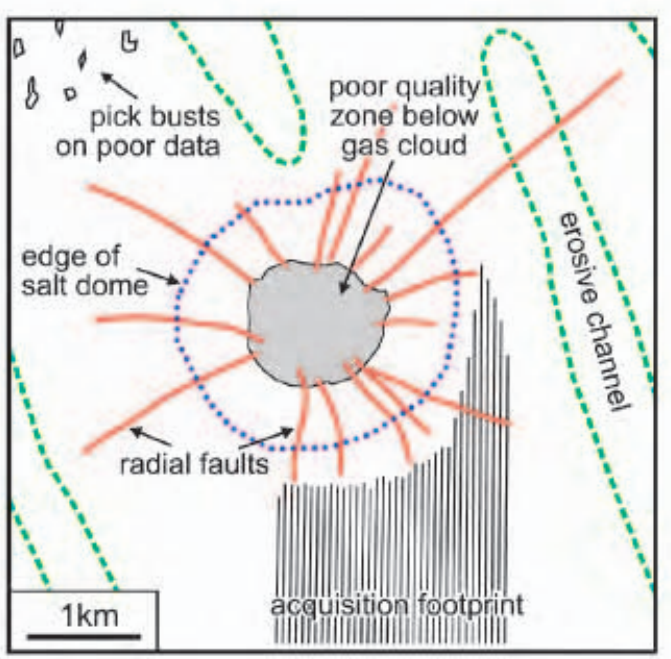

(e)

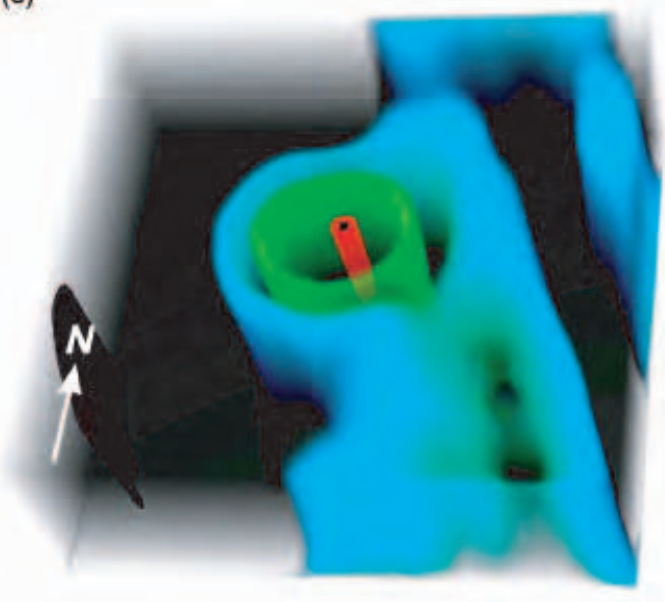

(C) 2004 Blackwell Publishing Ltd, Basin Research, 16, 183-197 
As before, the scale of each derived signal is defined by selecting a different standard deviation $\sigma$ for the Gaussian function. Each hierarchical layer in a stack represents convolution at a fixed scale, with the smallest scale at the bottom, and the largest at the top (Fig. 7c). This scale-space cube is analogous to the scale-space surface illustrated in Fig. 3e.

\section{Blob-feature detection}

To define dominant features in the cube, we apply blobfeature detection. This is analogous to the identification of zero-crossings of curvature discussed earlier in relation to natural scales in cross sections. Hay et al. (2002) describe this procedure and terminology in detail; a summary of the key steps is presented here. The primary objective of this nonlinear feature detection approach is to link structures at different scales in scale-space to higher-order objects called 'scale-space blobs', and to extract significant features based on their appearance and persistence through scales. The main features that arise at each scale within a stack are smooth regions, which are brighter or darker than the background and stand out from their surroundings. These regions are termed 'grey-level blobs' (Fig. 7d). When blobs are evaluated as a volumetric structure within a stack, it becomes apparent that some structures visually persist through scale, while others disappear (Fig. 7e). Therefore, an important premise of scale-space is that blob-like structures that persist in scale-space are likely to correspond to significant structures in the initial image, and thus in the real-world scene or structure. Grey-level blobs at each scale in the stack are treated as objects with extent both in 2D space $(x, y)$ and in grey level (height attribute) - thus 3D (and the structures that are persistent through scale are therefore four-dimensional (4D)).

Grey-level blob delineation can also be described with a watershed analogy. At each scale in the stack, the image function of all blobs may be considered as a flooded 3D landscape (i.e. a watershed). As the water level gradually sinks, peaks appear. At some point, two different peaks become connected. The corresponding connection elevation levels are called the base level of the blob. They are used for delimiting the 2D spatial extent or 'region of support' of each blob, which is defined as a binary blob (Fig. 8). The actual technique for defining binary blobs involves convolving the original image with the Laplacian of Gaussian at different standard deviations, then identifying zero-cross- ings to give binary blobs at each scale. This filter combines smoothing and calculation of second derivative (Eqn. (3)).

$L o G(x, y)=-\frac{1}{\pi \sigma^{4}}\left[1-\frac{x^{2}+y^{2}}{2 \sigma^{2}}\right] e^{-\left(\left(x^{2}+y^{2}\right) / 2 \sigma^{2}\right)}$

Figure $7 \mathrm{~d}$ compares zero-crossings calculated in this way with the corresponding $\sigma=200$ greyscale image. This shows that (particularly at large scale) our implementation is only partially successful in matching greyscale structure. Optimizing this step is the subject of future work. Nonetheless, the binary blobs shown in Fig. 8 highlight many of the features identified in the initial structural sketch (Fig. 7b). This is still, however, an arbitrary sampling of the scale-space continuum; the next stage is extraction of significant elements from this data set.

$2 \mathrm{D}$ binary blobs at all scales are combined within a new stack to create 3D 'hyper-blobs' (Fig. 9). Individual hyperblobs can be characterised by one or more of four primary topological elements or 'bifurcation events' (Fig. 10). The ability to define these scale-space-events represents a critical component of scale-space analysis, as scales between bifurcations are linked together forming the lifetime $\left(L \sigma_{n}\right)$ and topological structure of individual scale-space blobs. Once the lifetime of each scale-space blob is established, the integrated normalised $4 \mathrm{D}$ volume $(x, y, z, \sigma)$ of each scale-space blob is defined. But since blob behaviour is strongly dependent upon image structure, it is possible that an expected image behaviour may exist that may or may not relate to significant multiscale structure - when evaluated through scale, even noise has structure (Hay et al., 2002). To allow for this, statistics that characterise noise are extracted from a large number of stacks made from random images. The resulting normalised volumes are then ranked from the highest to the lowest based on the size of their integrated 4D volume, and a user determined number of 'significant' scale-space blobs are extracted from this set. For each of the selected hyper-blobs, the scale $(\sigma)$ representing the maximum 3D grey-level blob volume $(x, y, z)$ is extracted. From these layers, the $2 \mathrm{D}$ spatial support (i.e., binary blob) is identified and related back to the corresponding structures in the image for viewing and further interpretation (Fig. 11). So, based on the underlying initial premise, 4D scale-space blobs are simplified to 3D grey-level blobs, which are further simplified to their 2D support region $(x, y)$, and then to their corresponding real-world object in the original image.

Fig. 7. Generation of a scale-space cube from 3D seismic interpretation. (a) $30 \mathrm{~km}^{2}$ patch of seismic interpretation of a surface within the Tertiary of the central North Sea. The surface lies at a depth of about $300 \mathrm{~m}$ below the seabed. Colourbar indicates elevation (red high, blue low). Sun shaded to emphasise edges. North is up. The surface is mapped at a grid spacing of $25 \mathrm{~m}$. (b) Sketch interpretation of main features in surface. Some pervasive features (pick busts and acquisition footprint) are only annotated in a small part of the sketch for clarity. (c) Scale-space stack (cube) created from the surface in part(a). The height (z-axis) in (a) is rendered as a greyscale attribute to form the initial image, and the imaged is resampled at a grid spacing of $100 \mathrm{~m}$. Each layer is smoothed by a Gaussian kernel of increasing scale $\sigma$. (d) Slice through scale-space cube showing grey-level blobs at $\sigma=200$. Rendered in 16-bit greyscale to emphasise blob shape. Overlay of polygons shows corresponding binary blob edges derived by Laplacian of Gaussian filtering at the same scale (see Fig. 8). (e) Scale-space cube rendered semi-transparent to show grey-level blobs that persist in scale. 

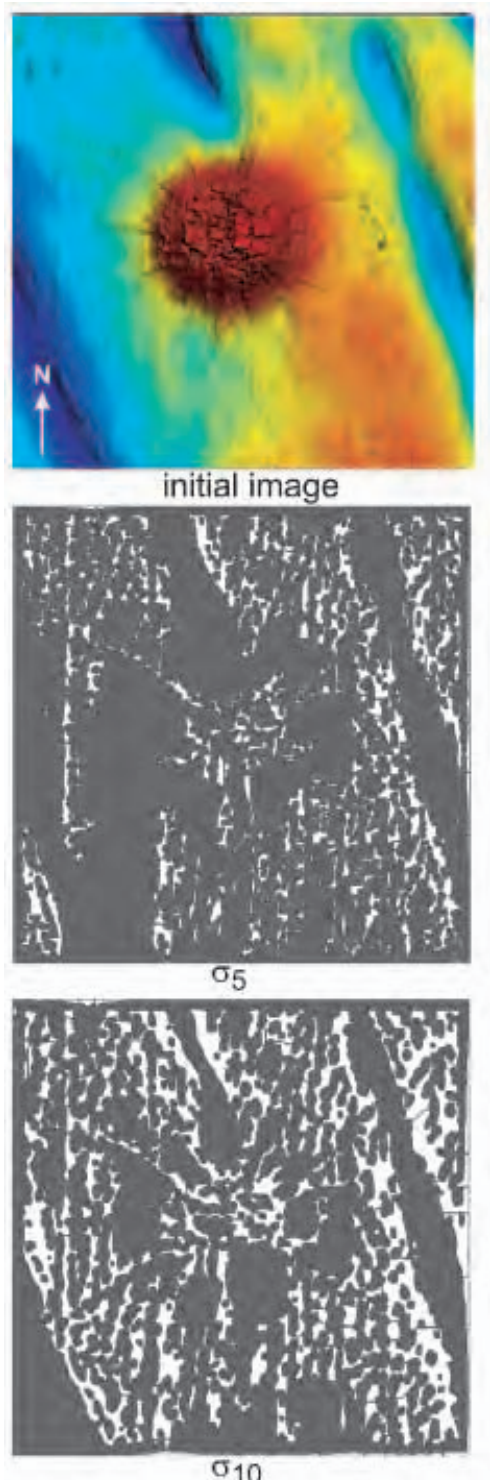
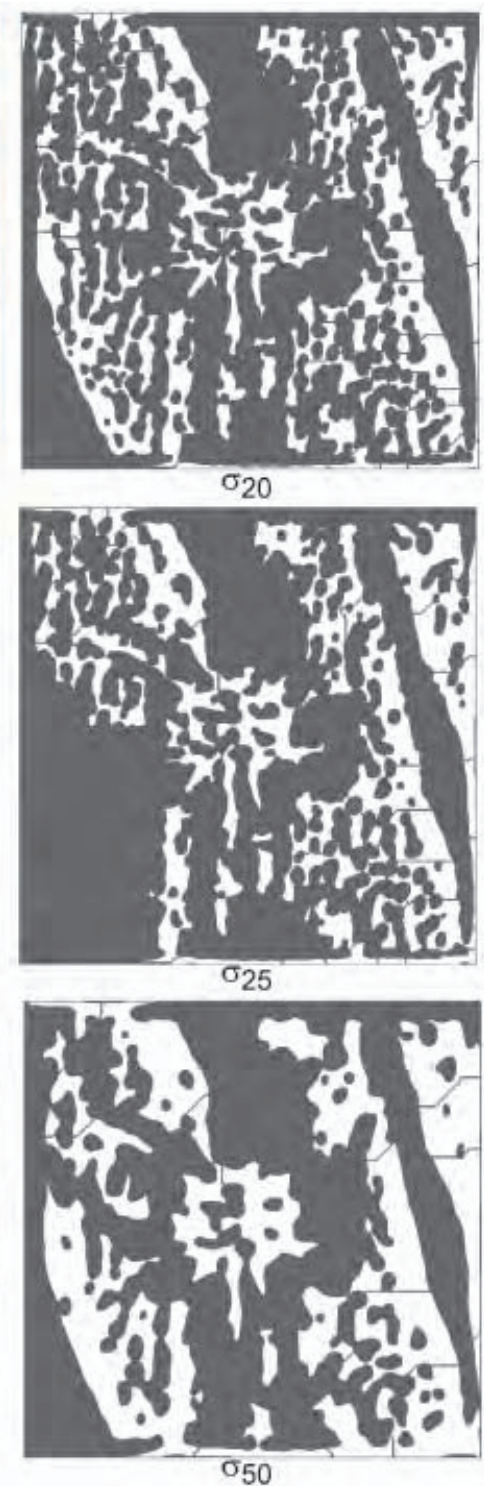
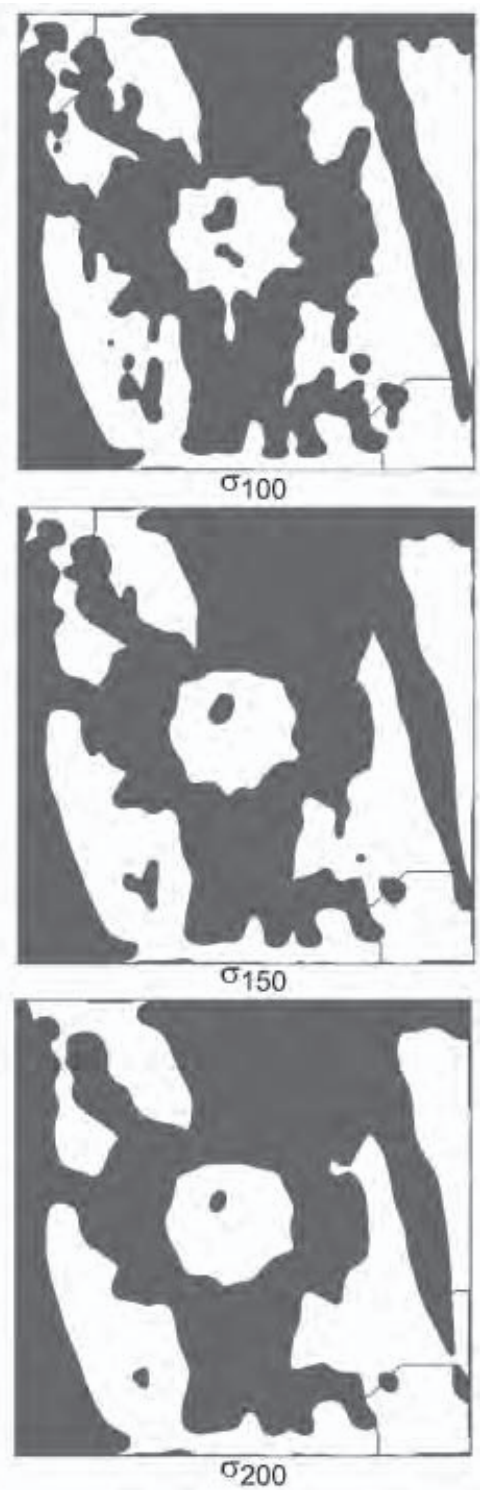

Fig. 8. Binary blobs derived by Laplacian of Gaussian filtering of the intial greyscale image at a range of scales, compared with the source seismic interpretation. The binary blobs highlight different features at different scales.

\section{Discussion of blob-feature detection}

This analysis yielded 4635 hyper-blobs, each represented by a $2 \mathrm{D}$ support region polygon. This represents automatic selection of $2 \mathrm{D}$ blobs corresponding to features that were arbitrarily intersected in the scale slices shown in Fig. 8. Visual appraisal of this large number of features is a substantial task in itself. Figure 1la shows 200 of the most significant blobs, rendered as unfilled 2D support region polygons. Even this small proportion (4\% of the total identified) is difficult to make sense of in a conventional map display. For clarity, in this short discussion the number is further reduced in Fig. 11b. Strengths and weaknesses of this approach are clear. The method has identified smallscale features (pick busts), and large-scale features (salt dome and erosive channels). On the other hand, narrow linear features such as the radial faults and the seismic acquisition footprint have not been identified as discrete ele- ments. Furthermore, some large features are only partially identified, for example the channel in the north of the area. There are also numerous circular features of approximately 500-m diameter that are not obvious in the original data set (Fig. 7a) - it is unclear if these are artifacts of the process or if they are a subtle geological phenomenon such as incipient polygonal faults. It is, however, encouraging that structures over a range of scales have been identified by this automatic procedure, but further work is evidently required to simplify the output of the feature detector.

\section{SUMMARY}

The density and extent of data acquired in seismic reflection surveys allows multiscale structural analysis. A number of possible methods for deriving scale-space and detecting structures within it have been reviewed - some 


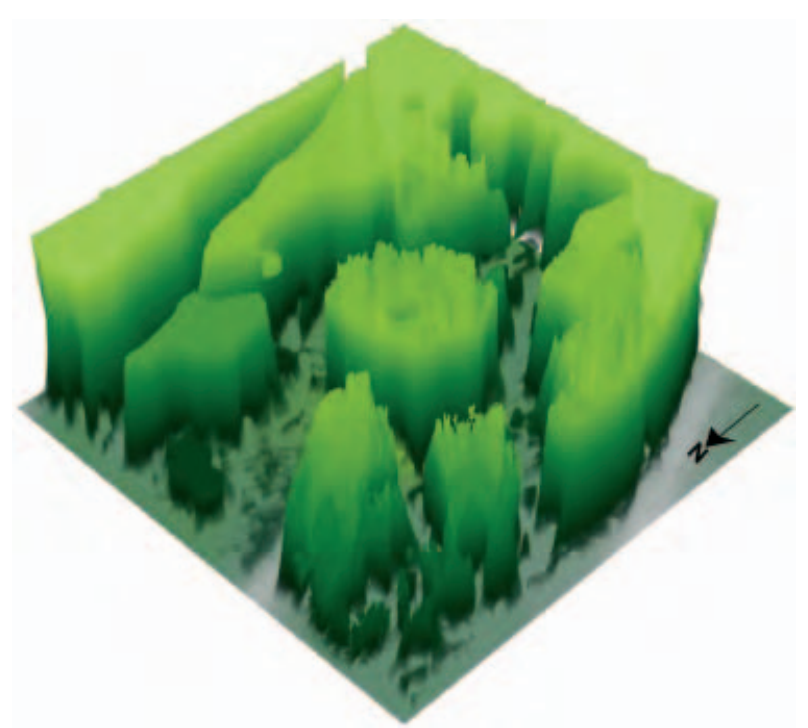

Fig. 9. Hypercube made in a manner similar to the scale-space cube shown in Fig. 7, but using binary blob masks, a selection of which are shown in Fig. 8 .

are standard techniques used by seismic interpreters, others are introduced from disciplines outwith geoscience. Introduction of some new terminology has been difficult to avoid, but minimised. Moving average filtering to remove seismic noise is common practice in the oil industry, but it is shown here that Gaussian filtering is more robust. A Gaussian filtering method of generating scale-space fulfils key criteria for multiscale analysis of geological surfaces identified at the start of this paper - it operates on the basis of no a priori knowledge of the scale and form of the geological structure, the derived scalespace can isolate localised features and the technique is applicable in 2D (i.e. sections) and 3D (surfaces). Feature detection in scale-space associated with sections and surfaces has been demonstrated using thresholding methods based on zero-crossings of curvature and second derivative (Laplacian) of surface elevation. The feature detectors tested here have been moderately successful at automatically identifying multiscale structure, but the results can be regarded as promising and represent a starting point for further investigation.

A relatively simple workflow for multiscale structure of a gridded surface that can be recommended on the basis of the work presented here is:

(1) Derive natural scales from a representative 2D section through the area of interest.

(2) Smooth the gridded surface using isotropic Gaussian kernels corresponding to these natural scales.

(3) Option to use high-order natural-scale surfaces to remove long wavelength curvature from low order natural scale surfaces.

(4) Further structural analysis, e.g. curvature analysis (Lisle, 1994; Stewart \& Podolski, 1998; Belfield, 2000; Bergbauer \& Pollard, 2003)

Use of bespoke curvature analysis methods after multiscale filtering as suggested in step 4 would yield anisotropic structure, such as periclinal folds, even though the filters that generate the surfaces are isotropic. Anisotropic Gaussian filters are available (e.g. Geusebroek et al., 2003), but variation in both anisotropy and orientation would multiply computer time and blob-feature detection complexity such that an automated method on this basis is difficult to envisage at this time.

Multiscale analysis using the methods presented in this paper, or analogous procedures (e.g. Bergbauer et al., 2003), are appropriate for addressing the structural geology questions identified at the start of this paper. Complete answers to these questions is beyond the scope of this paper, but some comments can be offered:

- How can superimposed geological structures of different scales be mapped and measured? Natural-scale analysis and scale-space topology could be used.

- To what extent are outcrop-scale rock properties like fracture permeability determined by larger scale struc-

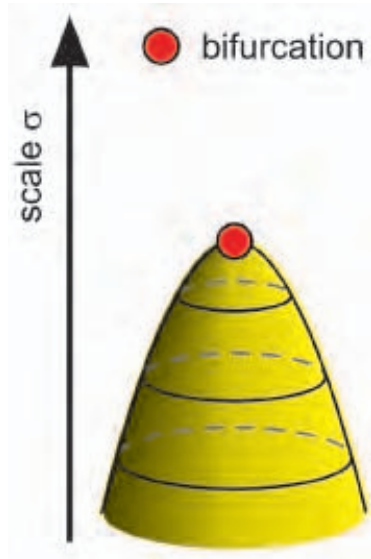

Annihilation

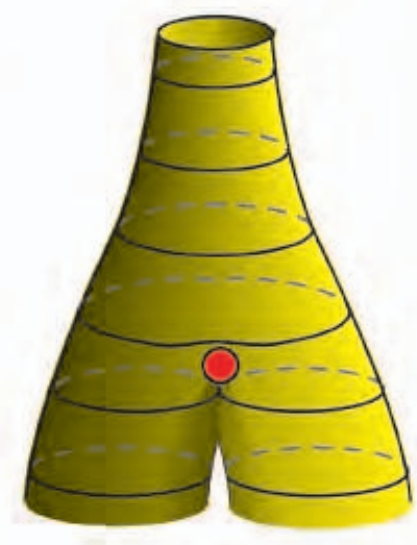

Merge

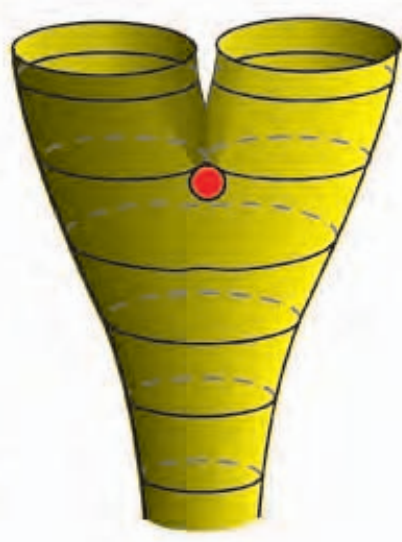

Split

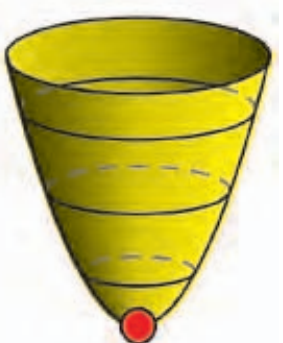

Creation

Fig. 10. Topological elements within scale-space, defined in relation to an increase in scale: Annihilation - one blob disappears, Merge - two blobs merge into one, Split - one blob splits into two, Creation - one new blob appears. 
(a)

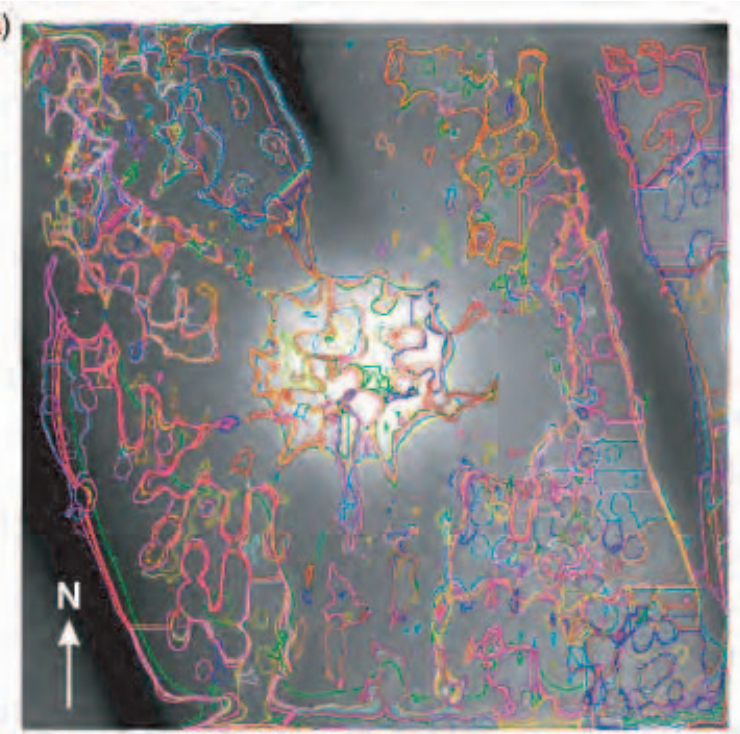

(b)

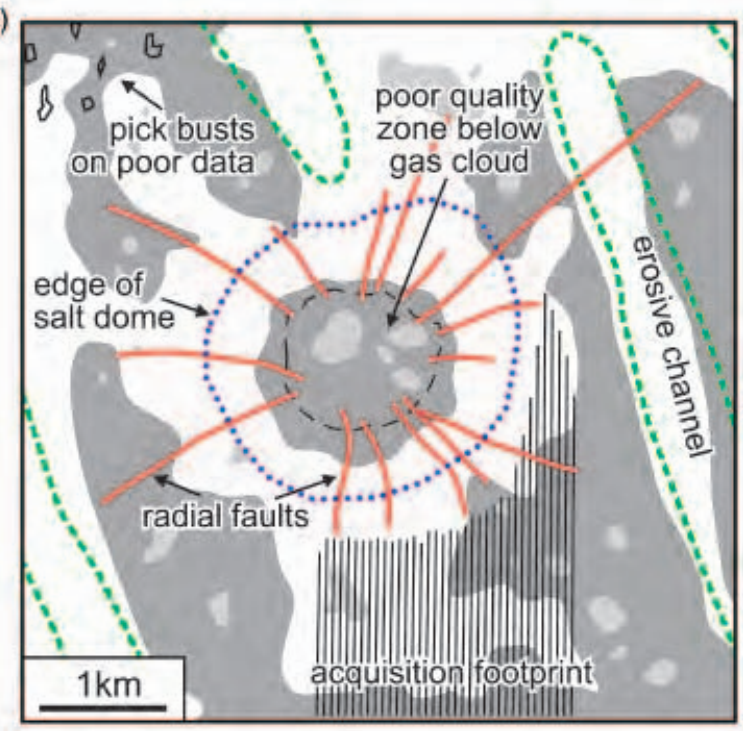

Fig. 11. Selection of 2D polygons or support regions, corresponding to $4 \mathrm{D}$ hyper-blobs identified as being significantly different from noise. (a) 200 of the most significant support regions overlaid on the $\sigma=200$ derivation of the original image. (b) User-defined 'top thirty' support regions (large ones shaded darker) compared with interpretation of the initial image.

tures that may be unseen at outcrop or in the wellbore? Structures or structural domains identified in scalespace are candidates for controlling widespread fracture sets.

- Should fold-classification schemes be qualified with scale-dependent terms? The examples presented in this paper strongly indicate that whether a fold is perceived or not depends entirely on the scale of observation, so a measure of fold scale should always accompany classification that is otherwise based on fold style or geometry. Scale-space analysis could underpin a novel, automated method of fold mapping and classification.
- Are interference and parasitic fold patterns end-members of a continuum? This could be the case in geometric, if not genetic, terms.

Possible applications of these methods in a hydrocarbon production context are indicated above; specific examples include automated removal of data noise from seismic interpretation and mapping of tectonically influenced reservoir quality domains.

\section{ACKNOWLEDGMENTS}

The opinions expressed here are solely those of the authors and not necessarily those of BP or TRACS International. The paper benefited from reviews by R. Lisle and S. Bergbauer. H. Macintyre and R. Hardy are thanked for bringing key work to the authors' attention.

\section{REFERENCES}

Babaud, J., Witkin, A.P., Baudin, M. \& Duda, R.O. (1986) Uniqueness of the Gaussian kernel for scale-space filtering. IEEE Trans. Pattern Anal. Mach. Intell., 8(1), 26-33.

BELFIELD, W.C. (1998) Incorporating spatial distribution into stochastic modelling of fractures: multifractals and Lévystable statistics. 7. Struct. Geol., 20, 473-486.

Belfield, W.C. (2000) Predicting natural fracture distribution in reservoirs from 3D seismic estimates of structural curvature. SPE E-Library Paper No. 60298.

Bengtsson, A. \& EkLundh, J.O. (1991) Shape representation by multiscale contour approximation. IEEE Trans. Pattern Anal. Mach. Intell., 13(1), 85-93.

Bergbauer, S., Mukerji, T. \& Hennings, P. (2003) Improving curvature analyses of deformed horizons using scale-dependent filtering techniques. American Association of Petroleum Geologists Bulletin, 87(8), 1255-1272.

Bergbauer, S. \& Pollard, D.D. (2003) How to calculate normal curvatures of sampled geological surfaces. 7. Struct. Geol., 25, 277-289.

Bonnet, E., Bour, O., Odling, N.E., Davy, P., Main, I., CowIE, P. \& Beкоwitz, B. (2001) Scaling of fracture systems in geological media. Rev. Geophys., 39(3), 347-383.

Carter, D.C. (2003) 3-D seismic geomorphology: insights into fluvial reservoir performance, Widuri Field, Java Sea. Am. $A s-$ soc. Petrol. Geol. Bull., 87, 909-934.

Cowie, P.A., Sornette, D. \& Vanneste, C. (1995) Multifractal scaling properties of a growing fault population. Geophys. $\mathcal{F}$. Int., 122, 457-469.

Darrozes, J., Gaillot, P., Saint-Blanquat, M. \& Bouchez, J.L. (1997) Software for multi-scale image analysis: the normalized optimized anisotropic wavelet coefficient method. Comput. Geosci., 23(8), 889-895.

Desbrun, M., Meyer, M., Schröder, P. \& Barr, A.H. (1999) Implicit fairing of irregular meshes using diffusion and curvature flow. Proceedings SIGGRAPH '99, Computer Graphics Proceedings, Annual Conference Series, pp. 317-324. ACM Press, New York.

Ericsson, J.B., McKean, H.C. \& Hooper, R.J. (1998) Facies and curvature controlled 3D fracture models in a cretaceous carbonate reservoir, Arabian Gulf. In: Faulting, Fault Sealing 
and Fluid Flow in Hydrocarbon Reservoirs (Ed. by G. Jones, Q.J. Fisher \& R.J. Knipe), Geol. Soc. London Spec. Publ., 147, 299-312.

Finkelstein, A. \& Salesin, D.H. (1994) Multiresolution curves. In: Proceedings of SIGGRAPH 94 (Orlando, Florida, Fuly 24-29, 1994), Computer Graphics Proceedings, Annual Conference Series, ACM SIGGRAPH (Ed. by A. Glassner), pp. 261-268. ACM Press, New York.

Fleuty, M.J. (1964) The description of folds. Proc. Geol. Assoc., 75(4), 461-489.

Gallant, J.C. \& Hutchinson, M.F. (1997) Scale dependence in terrain analysis. Math. Comput. Simulation, 43(3-6), 313-321.

Gammaitoni, L., Hanggi, P., Jung, P. \& Marchesoni, F. (1998) Stochastic resonance. Rev. Modern Phys., 70(1), 223-287.

Geusebroek, J.M., Smeulders, A.W.M. \& van de Weijer, J. (2003) Fast anisotropic Gauss filtering. IEEE Trans. Image Process., 12(8), 938-943.

Gonzalez, R.C. \& Woods, R.E. (2002) Digital Image Processing, 2nd edn. Prentice-Hall, Englewoods Cliffs, NJ, 813pp.

Hart, B.S., Pearson, R. \& Rawling, G.C. (2002) 3-D seismic horizon-based approaches to fracture-swarm sweet spot definition in tight-gas reservoirs. Leading Edge, 21, 28-35.

Hay, G.J., Blaschke, T., Marceau, D.J. \& Bouchard, A. (2003) A comparison of three image-object methods for the multiscale analysis of landscape structure. Photogrammetric Eng. Remote Sensing, 57, 327-345.

Hay, G.J., Dubé, P., Bouchard, A. \& Marceau, D.J. (2002) A scale-space primer for exploring and quantifying complex landscapes. Ecol. Modelling, 153(1-2), 27-49.

Hay, G.J., Marceau, D.J., Dubé, P. \& Bouchard, A. (2001) A multiscale framework for landscape analysis: object-specific analysis and upscaling. Landscape Ecol., 16(6), 471-490.

KingSBury, N. (1999) Image processing with complex wavelets. Philos. Trans. R. Soc. London, Ser. A:Math. Phys. Eng. Sci., 357(1760), 2543-2560.

Lindeberg, T. (1994) Scale space theory: a basic tool for analysing structures at different scales. 7. Appl. Statist., 21, $224-270$.

Lindeberg, T. (1999) Principles for automatic scale selection. In: Handbook on Computer Vision and Applications, Vol. 2 (Ed. by B. Jähne, H. Haußecker \& P. Geißler), pp. 239-274. Academic Press, Boston, MA.

LisLe, R.J. (1994) Detection of zones of abnormal strains in structures using Gaussian curvature analysis. Am. Assoc. Petrol. Geol. Bull., 78, 1811-1819.

Lowe, D.G. (1989) Organization of smooth image curves at multiple scales. Int. 7. Comput. Vision, 3(2), 119-130.

Marceau, D.J. (1999) The scale issue in the social and natural sciences. Can. 7. Remote Sensing, 25(4), 347-356.

Marceau, D.J. \& Hay, G.J. (1999) Remote sensing contributions to the scale issue. Can. 7. Remote Sensing, 25(4), 357-366.

Mokhtarian, F. \& Mackworth, A.K. (1992) A theory of multiscale, curvature-based shape representation for planar curves. IEEE Trans. Pattern Anal. Mach. Intell., 14(8), 789-805.

Mokhtarian, F., Khalili, N. \& Yuen, P. (2002) Estimation of error in curvature computation on multi-scale free-form surfaces. Int. 7. Comput. Vision, 48(2), 131-149.

Pike, R.J. \& Rozema, W.J. (1975) Spectral analysis of landforms. Ann. Assoc.Am. Geogr., 65(4), 499-516.
Ramsay, J.G. (1967) Folding and Fracturing of Rocks. McGraw-Hill, New York, 568pp.

Ramsay, J.G. \& Huber, M.I. (1987) The Techniques of Modern Structural Geology, Vol. 2: Folds and Fractures. Academic Press Ltd, London, 700pp.

RoBerts, A. (2001) Curvature attributes and their application to 3D interpreted horizons. First Break, 19, 85-100.

Rosin, P.L. (1992) Representing curves at their natural scales. Pattern Recognition, 25(11), 1315-1325.

Rosin, P.L. (1998) Determining local natural scales of curves. Pattern Recognition Lett., 19(1), 63-75.

Sajda, P., Laine, A. \& Zeevi, Y. (2002) Multi-resolution and wavelet representations for identifying signatures of disease. $D i$. Markers, 18, 339-363.

SLutzKy, E. (1937) The summation of random causes as the source of cyclic processes. Econometrica, 5, 105-135.

Stabler, C.L. (1968) Simplified Fourier analysis of fold shapes. Tectonophysics, 6, 343-350.

Stewart, S.A. \& Podolski, R. (1998) Curvature analysis of gridded geological surfaces. In: Structural Geology in Reservoir Characterisation (Ed. by M.P. Coward, T.S. Daltaban \& H. Johnson), Geol. Soc. London, Spec. Publ., 127, 133-147.

STEWART, S.A. \& Wynn, T.J. (2000) Mapping spatial variation in rock properties in relationship to spectral curvature. Geology, 28(8), 691-694.

Stowe, C.W. (1988) Application of Fourier analysis for computer representation of fold shapes. Tectonophysics, 156, 303-311.

Tearpock, D.J. \& Bischke, R.E. (2002) Applied Subsurface Geological Mapping with Structural Methods, 2nd edn. Prentice-Hall, Englewood Cliffs, NJ, 864pp.

Telford, W.M., Geldart, L.P., Sheriff, R.E. \& Keys, D.A. (1976) Applied Geophysics. Cambridge University Press, Cambridge, $860 \mathrm{pp}$.

Veneziano, D. \& Iacobellis, V. (1999) Self-similarity and multifractality of topographic surfaces at at basin and subbasin scales. 7. Geophys. Res., 104, 12797-12812.

Weickert, J. (1997) A review of nonlinear diffusional filtering. In: Scale-space Theory in Computer Vision, Lecture Notes in Computer Science, Vol. 1252 (Ed. by B. ter Haar Romeny, L. Florack, J. Koenderink \& M. Viergever), pp. 3-28. Springer, Berlin.

WitKIN, A.P. (1984) Scale space filtering: a new approach to multi-scale description. In: Image Understanding 1984 (Ed. by S. Ullman \& W. Richards), pp. 79-95. Ablex, Norwood, NJ.

Wood, J. (1996) Scale-based characterisation of digital elevation models. In: Innovations in GIS 3 (Selected Papers from the Third National Conference on GIS Research) (Ed. by D. Parker), pp. 163176. Taylor \& Francis, Bristol.

Xu, T.B., Moore, I.D. \& Gallant, J.C. (1993) Fractals, fractal dimensions and landscapes - a review. Geomorphology, 8(4), 245-262.

Zhang, B.L., Coggins, R., Jabri, M.A., Dersch, D. \& FlowER, B. (2001) Multiresolution forecasting for futures trading using wavelet decompositions. IEEE Trans. Neural Networks, 12(4), 765-775.

Manuscript accepted: 17 March 2004 